\title{
Sugar makes neutrophils RAGE: linking diabetes- associated hyperglycemia to thrombocytosis and platelet reactivity
}

\author{
Robert H. Lee ${ }^{1}$ and Wolfgang Bergmeier ${ }^{1,2}$ \\ 'McAllister Heart Institute and 2Department of Biochemistry and Biophysics, University of North Carolina at Chapel Hill, Chapel Hill, North Carolina, USA.
}

\begin{abstract}
Diabetes mellitus is associated with an increased risk for cardiovascular disease, but the link between hyperglycemia and atherothrombotic disease is not completely understood. Patients with diabetes often show hyporesponsiveness to antiplatelet therapies, and it has been suggested that hyperreactive reticulated platelets underlie this altered therapeutic response. In this issue of the $J C I$, Kraakman et al. uncover a previously unknown link between hyperglycemia and enhanced platelet production and reactivity. The authors demonstrate that high blood glucose levels trigger neutrophil release of $\mathrm{S} 100$ calcium-binding protein A8/A9 (S100A8/ A9), which binds to the receptor for advanced glycation end products (RAGE) on Kupffer cells, ultimately leading to increased thrombopoietin (TPO) production in the liver. TPO causes megakaryocyte proliferation and increased platelet production. This study demonstrates the importance of glycemic control and identifies potential therapeutic targets in the normalization of platelet numbers and function in diabetes.
\end{abstract}

\section{Introduction}

Diabetes mellitus is a disease characterized by an inability of the body to produce or respond to insulin, resulting in hyperglycemia. Type 1 diabetes (T1D) is caused by autoimmune destruction of insulin-producing pancreatic $\beta$ cells (also known as juvenile diabetes). Type 2 diabetes (T2D) results from progressive resistance to insulin, often found in older individuals, and is associated with excessive body weight, poor diet, and lack of exercise (also known as adult-onset diabetes). T2D accounts for approximately $90 \%$ of all diabetes cases, affecting almost $10 \%$ of the adult population (1). Uncontrolled diabetes results in various pathologies, including thrombotic complications such as myocardial infarction and stroke. The risk of cardiovascular disease (CVD) can persist even in diabetic patients whose blood glucose levels are controlled (2). But how is diabetes linked to an increased risk for CVD? The answer, at least to some extent, seems to lie in dysregulation of the number and function of platelets.

The main physiological role of platelets is in the prevention of blood loss at sites of vascular injury (hemostasis). To guard the integrity of the vasculature, platelets are equipped with specialized surface-expressed adhesion and agonist receptors and a unique intracellular signaling machinery, all of which are critical for adhesion and hemostatic plug formation under high shear stress conditions such as are found in arterioles and arteries. However, these activating mechanisms are highly sensitive and pose a risk of platelet hyperreactivity, which can lead to the development of atherosclerotic plaques (atheroprogression) and the thrombotic complications associated with plaque rupture (atherothrombosis). Under physiological conditions, platelet numbers are tightly controlled,

Related Article: p. 2133

Conflict of interest: The authors have declared that no conflict of interest exists.

Reference information: / Clin Invest. 2017;127(6):2040-2043. https://doi.org/10.1172/JCI94494.

and the peripheral platelet count reflects both the rate of platelet production and the clearance of platelets from the blood. Once released into the circulation by megakaryocytes (MKs) within the bone marrow, platelets circulate for approximately 10 days before getting cleared, primarily by phagocytes of the reticulo-endothelial system. Platelet generation (thrombopoiesis) depends on the proliferation and differentiation of MKs, which are driven by thrombopoietin (TPO) produced by various cell types, including marrow stromal cells and hepatocytes (3). Platelets newly released into the blood are larger in size, contain more mRNA ("reticulated"), and are more reactive to agonist stimulation than are older cells (4). These highly reactive reticulated platelets may be the key to understanding the enhanced risk for CVD and thrombotic events in diabetes.

It is well established that diabetes leads to significant changes in blood platelets, including increased mean platelet volume (MPV) and greater numbers of reticulated platelets (5), a phenotype often documented in T2D patients. Consistently, circulating platelets in diabetic patients are hyperreactive to agonist stimulation and are more likely to form aggregates with circulating leukocytes, thereby increasing inflammation in these patients (6). Thus, it is proposed that a high reticulated platelet count contributes to the high risk for thrombotic events in diabetic patients (Figure 1) and that normalization of platelet function could positively affect outcomes in these patients (7). Current strategies to prevent atherothrombosis include the use of inhibitors of cyclooxygenase-1 (aspirin) and inhibitors of the platelet ADP receptor P2Y12 such as clopidogrel, prasugrel, or ticagrelor. While effective in most individuals, significant pharmacological hyporesponsiveness to these inhibitors has been documented for various disease states, including diabetes. Therefore, it is critical 


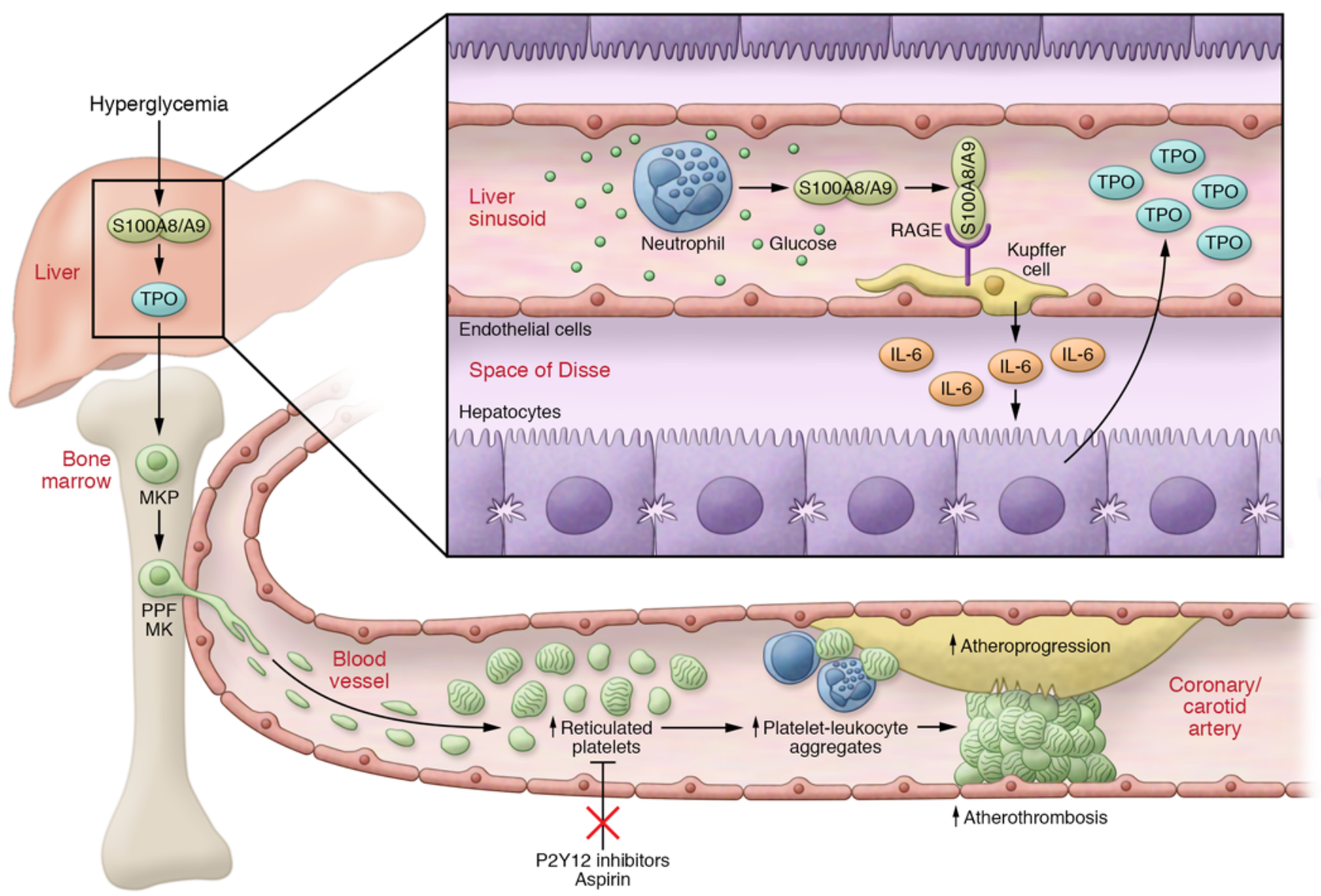

Figure 1. Neutrophil S100A8/A9 induces TPO production and reticulated thrombocytosis in hyperglycemia. In STZ-treated diabetic mice, hyperglycemia causes neutrophils to release S100A8/A9 into the blood. Binding of S100A8/A9 to Kupffer cell RAGE triggers IL-6 production, leading to TP0 secretion from hepatocytes. Increased platelet production results in greater numbers of reticulated platelets, which are associated with both atheroprogression and atherothrombosis in humans. In diabetes, higher numbers of reticulated platelets are also associated with altered responsiveness to antiplatelet therapies. MKP, megakaryocyte precursor; PPF, proplatelet formation; TPO, thrombopoietin; RAGE, receptor for advanced glycation end products.

to identify the mechanisms underlying enhanced reticulated platelet counts and platelet reactivity in diabetes to better tailor antiplatelet therapies for these patients.

In this issue, Kraakman and colleagues describe a novel link between hyperglycemia and increased reticulated platelet counts and thrombocytosis (8). Their elegant mechanistic studies identify various approaches with the potential to normalize platelet function in diabetes. Moreover, these studies highlight the complexities of metabolic perturbations on various organ and cell systems throughout the body.

\section{Neutrophils drive hepatic TPO production and thrombopoiesis in diabetes}

In mice, T1D can be modeled using treatment with the pancreatic $\beta$ cell toxin streptozotocin (STZ). Kraakman and colleagues demonstrate that STZ-treated diabetic mice exhibit robust thrombocytosis, a substantial increase in reticulated platelet numbers, upregulation of P-selectin on the platelet surface, and higher numbers of platelet-neutrophil and platelet-monocyte aggregates in the blood. In accordance with elevated platelet counts, diabetic mice have higher levels of plasma TPO. Under physiological conditions, hepatocytes are a major source for circulating TPO. Analysis of livers in STZ-treated mice uncovered greater numbers of resident macrophages (Kupffer cells). Additionally, Kupffer cells from STZ-treated animals produce more IL-6 than do Kupffer cells from normoglycemic mice. Kraakman et al. suggest that this local IL-6 production then induces TPO production in hepatocytes. Finally, the increase in Kupffer cell abundance and IL-6 expression is shown to be in response to neutrophil-derived $\mathrm{S} 100 \mathrm{cal}-$ cium-binding protein A8/A9 (S100A8/
A9, also known as myeloid-related protein 8/14) binding to the receptor for advanced glycation end products (RAGE) (Figure 1). Kraakman and colleagues thus connect diabetes-induced hyperglycemia to thrombocytosis and an increase in reticulated platelets, independently of any direct action of glucose on MK function and/or platelet survival.

Neutrophils are glucose-sensitive inflammatory cells and have previously been shown to secrete the proinflammatory protein S100A8/A9 under conditions of hyperglycemia (9). Plasma S100A8/A9 is a member of the large family of calcium-binding proteins that is predominantly found in the cytoplasm of blood cells and in plasma. Elevated plasma levels of S100A8/ A9 are considered a biomarker for chronic inflammatory disorders such as inflammatory bowel disease and rheumatoid arthritis (10). In addition, enhanced release of 
S100A8/A9 from neutrophils has been shown to modulate myelopoiesis (9). Kraakman et al. use a variety of genetic and therapeutic approaches to demonstrate the connection between hyperglycemia, neutrophil-derived S100A8/A9, and thrombocytosis. Thrombocytosis is normalized in STZ-treated mice lacking S100A9 (which also lack S100A8) or treated with an inhibitor of S100A8/A9 (ABR-215757). Moreover, depletion of circulating neutrophils in STZ-treated animals normalizes thrombocytosis. Consistent with these findings in mice, plasma S100A8/A9 levels are elevated in human $\mathrm{T} 2 \mathrm{D}$ patients (8) and in T1D patients with coronary artery disease (CAD) (9). Unfortunately, total and reticulated platelet numbers were not reported for the T1D patients. Finally, Kraakman and colleagues demonstrate that treatment with the S100A9 inhibitor ABR-215757 ameliorates diabetes-induced atheroprogression in STZ-treated mice. Altogether, these results confirm a role for S100A8/A9 in CVD and implicate these proteins in the regulation of hyperglycemia-induced reticulated thrombocytosis in mice.

\section{Remaining questions and future directions}

The studies by Kraakman et al. suggest an intriguing and novel mechanism for how hyperglycemia in diabetes patients is coupled to alterations in MK biology, leading to thrombocytosis and an increase in the reticulated platelet count. These studies provide important answers to questions regarding the molecular mechanisms that underlie altered platelet function in diabetes but also raise a number of important questions that should be addressed in the future. Arguably, the most important question is, how well do these studies in mice translate to humans with diabetes? Compared with the marked thrombocytosis observed in STZ-treated mice ( 2 -fold increase in platelet counts), patients with T1D do not typically present with thrombocytosis (11), and increases in platelet counts are variable in patients with T2D. Diabetic patients, however, do show a marked increase in reticulated platelet counts, even in the absence of thrombocytosis. For example, T2D patients in the Kraakman et al. study had approximately $3.2 \times 10^{10}$ reticulated platelets per liter of blood compared with approximately $1.4 \times$
$10^{10}$ reticulated platelets per liter of blood observed in healthy controls (8). Future studies should address whether TPO levels are also increased in patients and whether platelet survival is affected at all in diabetic patients and/or mice. Studies of myeloproliferative disease suggest that an increase in TPO signaling increases platelet production but also causes enhanced turnover of platelets in the circulation (12). An increased platelet turnover rate could explain why, unlike mice, patients with diabetes typically have high reticulated platelet counts in the absence of significant thrombocytosis.

Similar to STZ-treated mice, young T1D patients tend to be normolipidemic and have a normal or even below-average weight and BMI. Postprandial glycemic control has been shown to be effective in lowering the blood glucose levels of these patients, but, as suggested in the study by Kraakman and colleagues, a few short glycemic spikes per day may be enough to drive chronic thrombocytosis and increase the number of reticulated platelets. Thus, pediatric T1D patients may benefit greatly from therapies that target the S100A8/ A9/RAGE/IL-6 pathway. In contrast, T2D patients are older and often present with comorbidities such as obesity and metabolic disease. Hyperlipidemia also influences platelet production and function and thus may at least partially negate the positive effects of therapies that limit S100A8/A9/RAGE/IL-6 signaling (13, 14). It is also important to remember that S100A8/A9 and RAGE are not selective for each other and switch binding partners depending on the disease condition (15). Thus, more studies will be required to validate that the observed benefit of targeting S100A8/A9/RAGE in STZ-treated mice, a model for T1D, can lead to new therapies for T2D, which is the much more prevalent form of diabetes.

Another intriguing finding of the Kraakman et al. study is the role of neutrophil S100A8/A9 in the regulation of TPO levels and thus MK function. On the basis of the inhibitor studies in mice, Kraakman and colleagues propose that inhibition of neutrophil-derived S100A8/A9 could be used to normalize platelet counts and/or function and to reduce susceptibility to CVD in individuals with diabetes. This is not the first report to identify a connection between elevated S100A8/A9 levels and CVD. Using next-generation sequencing and biochemical techniques, Healy and colleagues previously demonstrated that S100A8/A9 levels in platelets markedly increase before acute ST-segment elevation myocardial infarction (STEMI) and that elevated plasma concentrations of S100A8/9 predict the risk of future cardiovascular events in healthy individuals (16). In the current study, Kraakman et al. provide evidence that neutrophils are the critical source of S100A8/9 in diabetic mice. However, while mouse platelets express little S100A8/9, human platelets do express substantial levels of the protein $(17,18)$. Thus, it is possible that platelet-derived S100A8/9 also contributes to altered thrombopoiesis in humans with diabetes.

Irrespective of how exactly thrombopoiesis is altered in diabetes, it seems clear that platelet hyperreactivity contributes to the increased risk of cardiovascular complications and that there is an inadequate response to antiplatelet therapy in many diabetic patients. It is controversial at the moment whether the latter is a reflection of increased platelet production and thus reduced drug exposure, or whether the lack of response to antiplatelet therapy is a consequence of platelet hyperreactivity in these patients $(19,20)$. A multitude of factors, including changes in endothelial cell function, platelet signaling, platelet ROS formation, and platelet receptor expression, have been suggested to contribute to the altered reactivity of diabetic platelets (21). At this point, however, we lack conclusive information about which of these mechanisms are most critical for altered platelet reactivity in patients with diabetes and how antiplatelet therapy could be improved to enhance efficacy in the treatment of diabetes. The findings by Kraakman and colleagues are so exciting, because they offer an alternative approach that instead targets altered thrombopoiesis in diabetes.

\section{Acknowledgments}

We thank Nigel Mackman for helpful discussions. This work was supported by grants from the National Heart, Lung, and Blood Institute (NHLBI), NIH (1R01HL130404 and P01HL120846, to W. Bergmeier) and (4T32HL007149, to R.H. Lee). 
Address correspondence to: Wolfgang Bergmeier, Department of Biochemistry and Biophysics, 120 Mason Farm Road, 3113 Genetic Medicine Building, Campus Box 7260, University of North Carolina, Chapel Hill, North Carolina 27599, USA. Phone: 919.962.7331; E-mail: bergmeie@ email.unc.edu.

1. Benjamin EJ, et al. Heart Disease and Stroke Statistics - 2017 Update: A Report From the American Heart Association. Circulation. 2017;135(10):e146-e603.

2. Mazzone T. Intensive glucose lowering and cardiovascular disease prevention in diabetes: reconciling the recent clinical trial data. Circulation. 2010;122(21):2201-2211.

3. Grozovsky R, Giannini S, Falet H, Hoffmeister KM. Novel mechanisms of platelet clearance and thrombopoietin regulation. Curr Opin Hematol. 2015;22(5):445-451.

4. Thompson CB, Eaton KA, Princiotta SM, Rushin CA, Valeri CR. Size dependent platelet subpopulations: relationship of platelet volume to ultrastructure, enzymatic activity, and function. $\mathrm{Br} \mathrm{J}$ Haematol. 1982;50(3):509-519.

5. Tschoepe D, et al. Large platelets circulate in an activated state in diabetes mellitus. Semin Thromb Hemost. 1991;17(4):433-438.

6. Kakouros N, Rade JJ, Kourliouros A, Resar JR. Platelet function in patients with diabetes melli- tus: from a theoretical to a practical perspective. Int J Endocrinol. 2011;2011:742719.

7. Verdoia M, et al. Impact of diabetes on immature platelets fraction and its relationship with platelet reactivity in patients receiving dual antiplatelet therapy. J Thromb Thrombolysis. 2016;42(2):245-253.

8. Kraakman MJ, et al. Neutrophil-derived S100 calcium-binding proteins A8/A9 promote reticulated thrombocytosis and atherogenesis in diabetes. JClin Invest. 2017;127(6):2133-2147.

9. Nagareddy PR, et al. Hyperglycemia promotes myelopoiesis and impairs the resolution of atherosclerosis. Cell Metab. 2013;17(5):695-708.

10. Pruenster M, Vogl T, Roth J, Sperandio M. S100A8/A9: From basic science to clinical application. Pharmacol Ther. 2016;167:120-131.

11. Malachowska B, et al. Altered platelets' morphological parameters in children with type 1 diabetes - a case-control study. BMC Endocr Disord. 2015;15:17.

12. Maugeri N, et al. Clearance of circulating activated platelets in polycythemia vera and essential thrombocythemia. Blood. 2011;118(12):3359-3366.

13. Murphy AJ, et al. Cholesterol efflux in megakaryocyte progenitors suppresses platelet production and thrombocytosis. Nat Med. 2013;19(5):586-594.

14. Calkin AC, et al. Reconstituted high-density lipoprotein attenuates platelet function in individuals with type 2 diabetes mellitus by promoting cholesterol efflux. Circulation.
2009;120(21):2095-2104.

15. Nagareddy PR, et al. Adipose tissue macrophages promote myelopoiesis and monocytosis in obesity. Cell Metab. 2014;19(5):821-835.

16. Healy AM, et al. Platelet expression profiling and clinical validation of myeloid-related protein-14 as a novel determinant of cardiovascular events. Circulation. 2006;113(19):2278-2284.

17. Rowley JW, et al. Genome-wide RNA-seq analysis of human and mouse platelet transcriptomes. Blood. 2011;118(14):e101-e111.

18. Burkhart JM, et al. The first comprehensive and quantitative analysis of human platelet protein composition allows the comparative analysis of structural and functional pathways. Blood. 2012;120(15):e73-e82.

19. Armstrong PC, et al. Newly formed reticulated platelets undermine pharmacokinetically short-lived antiplatelet therapies [published online ahead of print March 9, 2017]. Arterioscler Thromb Vasc Biol. https://doi.org/10.1161/ATVBAHA.116.308763.

20. Stratz C, et al. Impact of reticulated platelets on antiplatelet response to thienopyridines is independent of platelet turnover. Thromb Haemost. 2016;116(5):941-948.

21. Schneider DJ, Hardison RM, Lopes N, Sobel BE, Brooks MM, Pro-Thrombosis Ancillary Study Group. Association between increased platelet P-selectin expression and obesity in patients with type 2 diabetes: a BARI 2D (Bypass Angioplasty Revascularization Investigation 2 Diabetes) substudy. Diabetes Care. 2009;32(5):944-949. 